\title{
Major Bleeding Risk During Anticoagulation with Warfarin, Dabigatran, Apixaban, or Rivaroxaban in Patients with Nonvalvular Atrial Fibrillation
}

\author{
Gboyega Adeboyeje, MD, MS; Gosia Sylwestrzak, MA; John J. Barron, PharmD; \\ Jeff White, PharmD, MS; Alan Rosenberg, MD; Jacob Abarca, PharmD, MS; \\ Geoffrey Crawford, MD, MS; and Rita Redberg, MD, MSc
}

\begin{abstract}
BACKGROUND: The use of non-vitamin K oral anticoagulants (NOACs) has increased steadily following marketing approval; however, their relative safety in nonvalvular atrial fibrillation (NVAF) patients in real-world clinical practice remains unclear.
\end{abstract}

OBJECTIVE: To compare the risk of major bleeding during anticoagulation therapy between warfarin and NOACs.

METHODS: This retrospective cohort study analyzed administrative claims data on new NVAF users of warfarin, dabigatran, apixaban, or rivaroxaban in routine clinical care from November 2010 to February 2015 in a commercially insured population in the United States. The primary outcome was time to first major bleeding event requiring hospitalization. Patients were followed until discontinuation or switch of anticoagulants, health plan disenrollment, death, or end of study. All patient characteristics were balanced after propensity score inverse probability of treatment (IPT) weighting. Event rates by type of anticoagulant exposure were compared using IPT-weighted Cox proportional hazards models.

RESULTS: The study cohort comprised 44,057 patients who used warfarin $(n=23,431)$, dabigatran $(n=8,539)$, apixaban $(n=3,689)$, and rivaroxaban $(n=8,398)$. Overall mean (SD) age was $70(12)$ years, and $41 \%$ of the patients were women. A total of 2,337 major bleeding events occurred during 36,636.2 person-years of follow-up. The unadjusted rate of major bleeding with warfarin was 6.0 per 100 person-years versus 2.8 with dabigatran, 3.3 with apixban, and 5.0 with rivaroxaban. Relative to warfarin, major bleeding risk was lower with dabigatran $(\mathrm{HR}=0.67,95 \% \mathrm{Cl}=0.60$ 0.76 ) and apixaban ( $\mathrm{HR}=0.52,95 \% \mathrm{Cl}=0.41-0.67)$. Compared with rivaroxaban, major bleeding risk was also lower with dabigatran $(\mathrm{HR}=0.67$, $95 \% \mathrm{Cl}=0.58-0.78)$ and apixaban ( $\mathrm{HR}=0.52,95 \% \mathrm{Cl}=0.40-0.68)$. Major bleeding risk was similar for rivaroxaban and warfarin. Relative to apixaban, dabigatran was associated with a significantly higher risk of major gastrointestinal bleeding ( $\mathrm{HR}=1.43,95 \% \mathrm{Cl}=1.09-1.88)$.

CONCLUSIONS: Study results were consistent with safety findings from pivotal clinical trials comparing NOACs with warfarin and added the perspective of a large real-world observational study that compared bleeding risks associated with NOACs during anticoagulation therapy. Apixaban and dabigatran were associated with lower major bleeding risk compared with warfarin or rivaroxaban; however, apixaban had a lower risk of major gastrointestinal bleeding than dabigatran. These findings can help inform the choice of an optimal agent, which must balance effectiveness and bleeding risk in complex patients.

J Manag Care Spec Pharm. 2017;23(9):968-78

Copyright $\odot 2017$, Academy of Managed Care Pharmacy. All rights reserved.

\section{What is already known about this subject}

Clinical trial results have demonstrated the efficacy and safety of non-vitamin K oral anticoagulants (NOACs) relative to warfarin for stroke prevention in nonvalvular atrial fibrillation (NVAF) patients.

Concerns persist regarding the real-world safety of NOACs.

\section{What this study adds}

This study examined major bleeding risk among NVAF patients using NOACs during anticoagulation therapy.

Relative to warfarin, dabigatran and apixaban were associated with a 33\% lower major bleeding risk, while dabigatran and apixaban were associated with a $48 \%$ lower risk of major bleeding compared with rivaroxaban.

Apixaban was associated with a lower risk of major gastrointestinal bleeding than dabigatran.

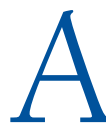
trial fibrillation is estimated to be responsible for approximately $15 \%-20 \%$ of all strokes. ${ }^{1}$ Long-term anticoagulation with vitamin $\mathrm{K}$ antagonists (e.g., warfarin) is effective for stroke prevention, but patients face a significant risk of major bleeding, and therapeutic effectiveness is complicated by dosing adjustments and dietary compliance..$^{2-4}$ Patients who bleed often discontinue anticoagulation so are at a higher risk of thromboembolism..$^{2-4}$

In recent years, the approval of several non-vitamin $\mathrm{K}$ oral anticoagulants (NOACs) expanded the therapeutic options for long-term anticoagulation in atrial fibrillation. NOAC agents (i.e., dabigatran, rivaroxaban, and apixaban) have compared favorably with warfarin in phase 3 trials with patients with nonvalvular atrial fibrillation (NVAF) $)^{5-7}$ and in a few observational studies. ${ }^{8-12}$ In addition, fewer drug-food or drug-drug interactions make NOACs appealing warfarin alternatives for stroke prophylaxis in atrial fibrillation..$^{2-4}$

NOACs accounted for about $62 \%$ of new prescriptions of oral anticoagulants in a 2014 report. ${ }^{13}$ Nonetheless, concerns persist regarding the lack of long-term experience with NOAC anticoagulation and effective management strategies in the 
Major Bleeding Risk During Anticoagulation with Warfarin, Dabigatran,

Apixaban, or Rivaroxaban in Patients with Nonvalvular Atrial Fibrillation

FIGURE 1 Cohort Definition Flow Diagram

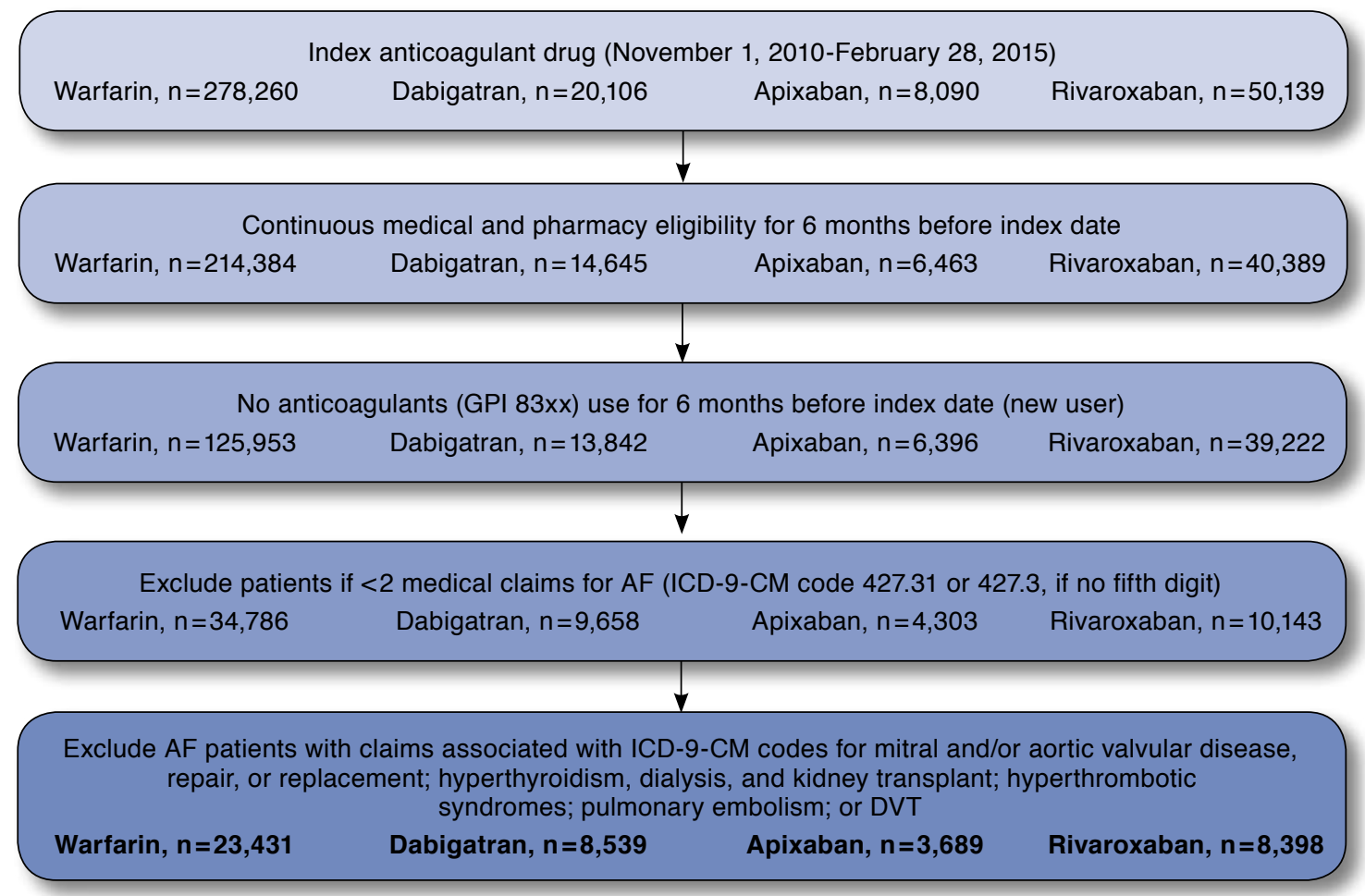

Note: Approval dates for stroke prevention NOACs to use in nonvalvular atrial fibrillation: dabigatran, October 2010; rivaroxaban, November 2011 (DVT prevention approval was July 2011); and apixaban, December 2012.

AF = atrial fibrillation; DVT = deep vein thrombosis; GPI = Generic Product Indicator; ICD-9-CM=International Classification of Diseases, Ninth Revision, Clinical Modification; NOAC=non-vitamin Koral anticoagulant.

event of major bleeding. The limited number of studies on the real-world safety of NOACs have reported conflicting findings. Whereas an analysis of U.S. Medicare data-covering beneficiaries who are typically aged 65 years and older-found a significantly higher risk of major bleeding and gastrointestinal bleeding with dabigatran compared with warfarin, ${ }^{14}$ another study reported no difference in the risk of gastrointestinal bleeding with dabigatran or rivaroxaban compared with warfarin in younger, commercially insured NVAF patients. ${ }^{15}$

In a large retrospective study of Medicare patients, Graham et al. (2016) reported that patients receiving rivaroxaban (20 mg once daily) were associated with statistically significant increases in major gastrointestinal bleeding, compared with patients who received dabigatran (150 mg twice daily). ${ }^{10}$ Noseworthy et al. (2016) reported lower bleeding risk among apixaban compared with dabigatran and rivaroxaban users, while rivaroxaban was associated with a higher bleeding risk compared with dabigatran. ${ }^{11}$ In a related study that compared NOACs with warfarin, Yao et al. (2016) reported that while apixaban and dabigatran were associated with lower risks of bleeding relative to warfarin, rivaroxaban and warfain appeared to have similar risks. ${ }^{12}$ In another large observational study, Deitelzweig et al. (2016) reported a higher risk of bleeding-related hospital readmissions among patients using rivaroxaban compared with those on apixaban, based on data from 2 large claims databases. ${ }^{9}$ Furthermore, Sussman et al. (2016) reported that patients treated with dabigatran in integrated networks had lower bleed-related health care utilization than those on warfarin. ${ }^{8}$

Meanwhile, a specific reversal agent has been approved for dabigatran, ${ }^{16}$ and factor Xa antidotes for apixaban- and rivaroxaban-related bleeding are in development, ${ }^{17,18}$ although experience with these antidotes in the management of NOACrelated major bleeding is limited.

Data on the relative safety of long-term anticoagulation with the newer agents in NVAF patients in routine care could inform the choice of anticoagulant. In this retrospective cohort study, we compared major bleeding outcomes for NVAF patients treated with warfarin, dabigatran, apixaban, or rivaroxaban. 


\section{Methods}

\section{Data Source}

We used medical and pharmacy claims data from the HealthCore Integrated Research Environment (HIRE) from November 1, 2009, through January 31, 2016. HIRE includes medical and pharmacy claims data for nearly 40 million members with commercial insurance across 14 U.S. regional health plans. All study data were kept anonymous to safeguard patient confidentiality; researchers only accessed a limited dataset, which was devoid of individual patient identifiers, in compliance with relevant provisions of the Health Insurance Portability and Accountability Act (HIPAA). This observational study, conducted under the research exception provisions of the HIPAA Privacy Rule 45 CFR 164.514(e), was granted an exemption from institutional review board review.

\section{Study Population}

New users of warfarin, dabigatran, apixaban, or rivaroxaban were identified from pharmacy claims. The index date was defined as the earliest prescription fill date of a comparator anticoagulant during the intake period (November 1, 2010, through February 28, 2015). Continuous health plan eligibility for 6 months was required before the index date. Patients were considered to be new users if they had no prescriptions for any anticoagulant in the 6-month period preceding their index dates. We identified patients with NVAF by the presence of $\geq 2$ medical claims (inpatient, emergency department, and outpatient) with International Classification of Diseases, Ninth Revision, Clinical Modification (ICD-9-CM) code 427.31 in the 6-month period before the index date. Patients with a diagnosis of cardiac valve disorders or valve replacement, hyperthyroidism, deep vein thrombosis, pulmonary embolism, kidney transplant, dialysis, or hyperthrombotic conditions were excluded (Figure 1). We followed each patient from the index date until a switch to a comparator anticoagulant, discontinuation of index anticoagulant (more than 45 days after 30-day prescription fill), health plan disenrollment, death, or end of study period, whichever occurred first.

\section{Outcomes and Covariates}

Outcomes. The primary outcome was time to the first major bleeding event leading to hospitalization. We identified major bleeding by an inpatient stay with a primary diagnosis of extracranial hemorrhage or any diagnosis of intracranial hemorrhage using previously validated algorithms. ${ }^{19-22}$ The use of inpatient ICD 9-CM codes to identify major bleeding has been shown to have a positive predictive value between $89 \%-98 \% .{ }^{21}$ In addition, we examined the risk of major gastrointestinal and intracranial bleeding separately.

Covariates. The type of anticoagulant exposure was our predictor variable of interest. We controlled for confounding due to age; sex; geographic region of residence; comorbidity burden by Deyo-Charlson Comorbidity Index ${ }^{23}$; stroke risk by $\mathrm{CHA}_{2} \mathrm{DS}_{2}$-VASc score (1 point each, except where noted, for history of congestive heart failure, hypertension, diabetes mellitus, previous stroke or transient ischemic attack or thromboembolism [2 points], vascular disease, sex category, and age 65 to 74 years (age $\geq 75$ is 2 points) ${ }^{24}$; bleeding risk by modified HAS-BLED concomitant score (1 point each for hypertension, abnormal liver function, abnormal renal function, stroke, bleed history, elderly [>65 years], drugs, e.g., antiplatelet or nonsteroidal anti-inflammatory drugs [NSAIDs] and alcohol use/abuse, if documented as diagnosis on medical claim, labile international normalized ratios [INR] not included, since these data were not available for all patients) ${ }^{25}$; history of chronic kidney disease; coronary artery disease; peripheral vascular disease; cancer; dyslipidemia; pericarditis; dementia; gait abnormalities; dizziness; diabetic and alcoholic neuropathy; esophageal varices; major trauma; coagulation defect factors; bleeding; and hospitalization before treatment initiation. We also assessed the use of antiplatelet agents, amiodarone, dronedarone, and any other antiarrhythmics, diuretics, vasopressors, steroids, progestin, estrogen, proton pump inhibitors (PPIs), NSAIDs, and COX-2 inhibitors in the baseline period (Appendix A, available in online article).

\section{Statistical Analysis}

Cox proportional hazards regression models with propensity score weighting were used to compare event rates by the type of anticoagulant exposure. ${ }^{26,27}$ In survival models, propensity score weighting has the advantage of greater bias reduction relative to stratification on propensity scores or covariate adjustment with propensity scores and outperforms matching on the propensity scores in terms of precision (provides estimates with lower mean squared error when estimating the effect of treatment in the treated). ${ }^{26}$

The analysis was conducted in 2 stages. First, we estimated the probabilities of receiving each anticoagulant, including the previously mentioned covariates as predictors using a generalized logit model. ${ }^{27-29}$ Each patient received 4 predicted probabilities for each anticoagulant. We used the inverse of the probability of the treatment a patient actually received as propensity score weights. To reduce the variance of the resulting weights, we multiplied the weights by the marginal probability of being treated with the anticoagulant received. To assess the degree of overlap (i.e., whether each patient had a positive probability of receiving each anticoagulant under comparison), we compared the distribution of estimated propensity scores and weights of each treatment regardless of the treatment actually received, using separate box plots. We assessed balance in the distribution of baseline characteristics using a maximum absolute standardized difference (MASD) threshold of 0.10 for all possible pairs of treatment groups (i.e., 6 in all). An MASD of 0.10 or less indicates negligible difference between measured 
Major Bleeding Risk During Anticoagulation with Warfarin, Dabigatran,

Apixaban, or Rivaroxaban in Patients with Nonvalvular Atrial Fibrillation

\section{FIGURE 2 Bleeding Outcomes, Entire Cohort and Subgroups, and NOACs Versus Warfarin, After Propensity} Score Weighting

\begin{tabular}{|c|c|c|c|c|c|c|c|c|}
\hline \multirow[b]{2}{*}{ Outcome } & \multicolumn{3}{|c|}{ Dabigatran vs. Warfarin } & \multicolumn{3}{|c|}{ Apixaban vs. Warfarin } & \multicolumn{2}{|c|}{ Rivaroxaban vs. Warfarin } \\
\hline & \multicolumn{2}{|r|}{$\begin{array}{c}\text { Favors } \\
\text { dabigatran }\end{array}$} & $\begin{array}{c}\text { Favors } \\
\text { warfarin }\end{array}$ & \multicolumn{2}{|r|}{$\begin{array}{c}\text { Favors } \\
\text { apixaban }\end{array}$} & $\begin{array}{c}\text { Favors } \\
\text { warfarin }\end{array}$ & $\begin{array}{c}\text { Favors } \\
\text { rivaroxaban }\end{array}$ & $\begin{array}{c}\text { Favors } \\
\text { warfarin }\end{array}$ \\
\hline Major bleeding events ${ }^{a}$ & $\begin{array}{c}0.67 \\
(0.60-0.76)\end{array}$ & + & & $\begin{array}{c}0.52 \\
(0.41-0.67) \\
\end{array}$ & -7 & & $\begin{array}{c}1.00 \\
(0.89-1.12)\end{array}$ & \\
\hline Intracranial bleeding & $\begin{array}{c}0.47 \\
(0.35-0.65) \\
\end{array}$ & -1 & & $\begin{array}{c}0.83 \\
(0.52-1.34) \\
\end{array}$ & -1 & & $\begin{array}{c}0.74 \\
(0.54-1.00) \\
\end{array}$ & \\
\hline Major GI bleeding & $\begin{array}{c}1.17 \\
(1.04-1.32) \\
\end{array}$ & & - & $\begin{array}{c}0.82 \\
(0.63-1.06) \\
\end{array}$ & $\rightarrow$ & & $\begin{array}{c}1.00 \\
(0.87-1.16) \\
\end{array}$ & \\
\hline \multicolumn{9}{|c|}{ Subgroups (major bleeding events) } \\
\hline \multicolumn{9}{|c|}{ History of bleeding } \\
\hline No & $\begin{array}{c}0.65 \\
(0.57-0.74) \\
\end{array}$ & -7 & & $\begin{array}{c}0.56 \\
(0.43-0.74) \\
\end{array}$ & $\rightarrow-$ & & $\begin{array}{c}1.00 \\
(0.88-1.14) \\
\end{array}$ & \\
\hline Yes & $\begin{array}{c}0.79 \\
(0.62-1.01) \\
\end{array}$ & -1 & & $\begin{array}{c}0.38 \\
(0.20-0.72) \\
\end{array}$ & $\rightarrow$ & & $\begin{array}{c}1.01 \\
(0.79-1.30)\end{array}$ & \\
\hline \multicolumn{9}{|l|}{ Age, years } \\
\hline$<65$ & $\begin{array}{c}0.37 \\
(0.26-0.53) \\
\end{array}$ & 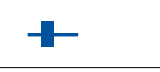 & & $\begin{array}{c}0.35 \\
(0.18-0.68) \\
\end{array}$ & $\rightarrow$ & & $\begin{array}{c}0.70 \\
(0.51-0.95) \\
\end{array}$ & \\
\hline $65-74$ & $\begin{array}{c}0.55 \\
(0.43-0.72) \\
\end{array}$ & -1 & & $\begin{array}{c}0.65 \\
(0.41-1.02) \\
\end{array}$ & & & $\begin{array}{c}0.97 \\
(0.71-1.23) \\
\end{array}$ & \\
\hline$\geq 75$ & $\begin{array}{c}0.83 \\
(0.72-0.96) \\
\end{array}$ & - & & $\begin{array}{c}0.55 \\
(0.39-0.77) \\
\end{array}$ & & & $\begin{array}{c}1.16 \\
(1.00-1.34) \\
\end{array}$ & $-1-$ \\
\hline \multicolumn{9}{|l|}{ HAS-BLED scoreb } \\
\hline$\leq 3$ & $\begin{array}{c}0.57 \\
(0.48-0.68) \\
\end{array}$ & 7 & & $\begin{array}{c}0.50 \\
(0.35-0.72) \\
\end{array}$ & -1 & & $\begin{array}{c}0.86 \\
(0.72-1.02) \\
\end{array}$ & \\
\hline$\geq 3$ & $\begin{array}{c}0.78 \\
(0.66-0.91) \\
\end{array}$ & & & $\begin{array}{c}0.54 \\
(0.38-0.77) \\
\end{array}$ & & & $\begin{array}{c}1.13 \\
(0.97-1.32) \\
\end{array}$ & + \\
\hline \multicolumn{9}{|l|}{ Hypertension } \\
\hline No & $\begin{array}{c}0.59 \\
(0.47-0.74) \\
\end{array}$ & - & & $\begin{array}{c}0.44 \\
(0.27-0.72) \\
\end{array}$ & -1 & & $\begin{array}{c}0.96 \\
(0.78-1.19) \\
\end{array}$ & \\
\hline Yes & $\begin{array}{c}0.71 \\
(0.62-0.82) \\
\end{array}$ & $t$ & & $\begin{array}{c}0.56 \\
(0.42-0.74) \\
\end{array}$ & & & $\begin{array}{c}1.01 \\
(0.88-1.16) \\
\end{array}$ & \\
\hline \multicolumn{9}{|l|}{ Diabetes mellitus } \\
\hline No & $\begin{array}{c}0.70 \\
(0.61-0.81) \\
\end{array}$ & t & & $\begin{array}{c}0.52 \\
(0.38-0.71) \\
\end{array}$ & -1 & & $\begin{array}{c}0.95 \\
(0.82-1.10) \\
\end{array}$ & \\
\hline Yes & $\begin{array}{c}0.62 \\
(0.50-0.77)\end{array}$ & $\rightarrow$ & & $\begin{array}{c}0.53 \\
(0.35-0.81) \\
\end{array}$ & & & $\begin{array}{c}1.09 \\
(0.90-1.32) \\
\end{array}$ & \\
\hline \multicolumn{9}{|l|}{ Heart failure } \\
\hline No & $\begin{array}{c}0.57 \\
(0.49-0.67) \\
\end{array}$ & - & & $\begin{array}{c}0.51 \\
(0.37-0.71) \\
\end{array}$ & -1 & & $\begin{array}{c}0.94 \\
(0.81-1.10) \\
\end{array}$ & \\
\hline Yes & $\begin{array}{c}0.85 \\
(0.71-1.01) \\
\end{array}$ & - & & $\begin{array}{c}0.54 \\
(0.37-0.81) \\
\end{array}$ & $r$ & & $\begin{array}{c}1.11 \\
(0.92-1.33) \\
\end{array}$ & \\
\hline \multicolumn{9}{|l|}{$\mathrm{CHA}_{2} \mathrm{DS}_{2}$-VASc score } \\
\hline 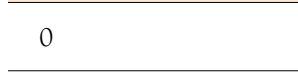 & $\begin{array}{c}0.37 \\
(0.14-1.03) \\
\end{array}$ & $\rightarrow$ & & $\begin{array}{c}0.55 \\
(0.11-2.80) \\
\end{array}$ & & $\longrightarrow$ & $\begin{array}{c}0.33 \\
(0.09-1.19) \\
\end{array}$ & \\
\hline 1 & $\begin{array}{c}0.39 \\
(0.21-0.72) \\
\end{array}$ & - & & $\begin{array}{c}0.23 \\
(0.05-0.98) \mathrm{d} \\
\end{array}$ & & & $\begin{array}{c}1.04 \\
(0.66-1.64) \\
\end{array}$ & \\
\hline$\geq 2$ & $\begin{array}{c}0.71 \\
(0.63-0.80) \\
\end{array}$ & $t$ & & $\begin{array}{c}0.55 \\
(0.42-0.71) \\
\end{array}$ & - & & $\begin{array}{c}1.02 \\
(0.91-1.15)\end{array}$ & $\vdash$ \\
\hline \multicolumn{9}{|l|}{ CKD 3 or worse } \\
\hline No & $\begin{array}{c}0.66 \\
(0.58-0.76) \\
\end{array}$ & t & & $\begin{array}{c}0.545 \\
(0.41-0.71) \\
\end{array}$ & - & & $\begin{array}{c}0.96 \\
(0.84-1.09) \\
\end{array}$ & \\
\hline Yes & $\begin{array}{c}0.75 \\
(0.56-1.01) \\
\end{array}$ & 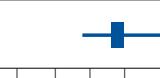 & & $\begin{array}{c}0.45 \\
(0.23-0.87) \\
\end{array}$ & $\longrightarrow$ & & $\begin{array}{c}1.28 \\
(0.98-1.69) \\
\end{array}$ & 1 \\
\hline & & $\begin{array}{lllll}0.2 & 0.4 & 0.6 & 0.8\end{array}$ & $\begin{array}{lllllll}0 & 1.2 & 1.4 & 1.6 & 1\end{array}$ & 0.0 & $\begin{array}{lllll} & 0.2 & 0.4 & 0.6 & 0.8\end{array}$ & $\begin{array}{lllll}1.2 & 1.4 & 1.6 & 1\end{array}$ & $\begin{array}{lllllll} & 0.0 & 0.2 & 0.4 & 0.6 & 0.8\end{array}$ & $\begin{array}{llll} & 1 & 1 & 1.2 \\
& 1.4 & 1.6\end{array}$ \\
\hline
\end{tabular}


Major Bleeding Risk During Anticoagulation with Warfarin, Dabigatran,

Apixaban, or Rivaroxaban in Patients with Nonvalvular Atrial Fibrillation

FIGURE 2 Bleeding Outcomes, Entire Cohort and Subgroups, and NOACs Versus Warfarin, After Propensity Score Weighting (continued)

\begin{tabular}{|c|c|c|c|c|c|c|c|c|}
\hline \multirow[b]{2}{*}{ Outcome } & \multicolumn{3}{|c|}{ Dabigatran vs. Warfarin } & \multicolumn{2}{|c|}{ Apixaban vs. Warfarin } & \multicolumn{3}{|c|}{ Rivaroxaban vs. Warfarin } \\
\hline & & $\begin{array}{c}\text { Favors } \\
\text { dabigatran }\end{array}$ & $\begin{array}{c}\text { Favors } \\
\text { warfarin }\end{array}$ & $\begin{array}{l}\text { Favors } \\
\text { apixaban }\end{array}$ & $\begin{array}{c}\text { Favors } \\
\text { warfarin }\end{array}$ & & $\begin{array}{c}\text { Favors } \\
\text { rivaroxaban }\end{array}$ & $\begin{array}{c}\text { Favors } \\
\text { warfarin }\end{array}$ \\
\hline \multicolumn{9}{|c|}{ Subgroups (major bleeding events) } \\
\hline \multicolumn{9}{|c|}{ NSAIDs } \\
\hline No & $\begin{array}{c}0.67 \\
(0.59-0.76) \\
\end{array}$ & + & & $\begin{array}{c}0.55 \\
(0.42-0.71) \\
\end{array}$ & & $\begin{array}{c}0.99 \\
(0.88-1.12) \\
\end{array}$ & & \\
\hline Yes & $\begin{array}{c}0.75 \\
(0.50-1.11) \\
\end{array}$ & - & & $\begin{array}{c}0.29 \\
(0.90-0.87)^{\mathrm{d}}\end{array}$ & & $\begin{array}{c}1.12 \\
(0.77-1.63)\end{array}$ & & - \\
\hline \multicolumn{9}{|c|}{ Platelet aggragation inhibitors } \\
\hline No & $\begin{array}{c}0.64 \\
(0.56-0.73) \\
\end{array}$ & $t$ & & $\begin{array}{c}0.51 \\
(0.38-0.67) \\
\end{array}$ & & $\begin{array}{c}0.93 \\
(0.81-1.06) \\
\end{array}$ & \multicolumn{2}{|c|}{-} \\
\hline Yes & $\begin{array}{c}0.85 \\
(0.65-1.10) \\
\end{array}$ & $\rightarrow$ & & $\begin{array}{c}0.56 \\
(0.32-0.98)^{\mathrm{d}} \\
\end{array}$ & & $\begin{array}{c}1.27 \\
(0.99-1.63) \\
\end{array}$ & & - \\
\hline & & 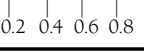 & $\begin{array}{lll}1.2 & 1.4 & 1.6 \\
\end{array}$ & $\begin{array}{llllll}0.0 & 0.2 & 0.4 & 0.6 & 0.8 \\
\end{array}$ & $\begin{array}{llll}1.2 & 1.4 & 1.6 & 1 \\
\end{array}$ & & $\begin{array}{llll}0.2 & 0.4 & 0.6 & 0.8 \\
\end{array}$ & 1.21 .41 .6 \\
\hline
\end{tabular}

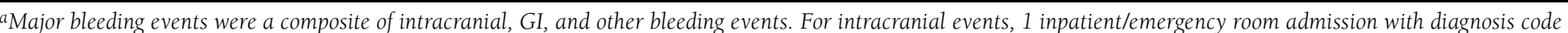
in any position on the claim; for other major bleeding events, primary diagnosis code was required.

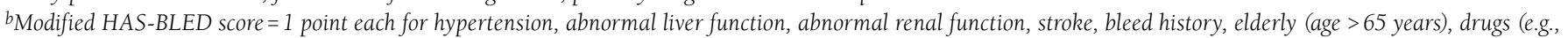

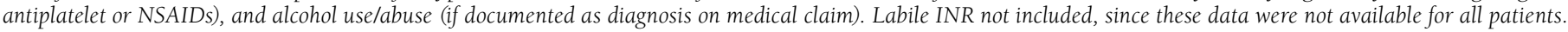

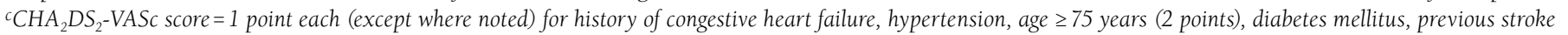
or TIA or thromboembolism (2 points), vascular disease, sex category and age 65-74 years.

dNot statistically significant after adjustment for multiple comparisons.

$C K D=$ chronic kidney disease; GI= gastrointestinal; INR=international normalized ratio; NOAC=non-vitamin Koral anticoagulant; NSAID = nonsteroidal anti-inflammatory drug; TIA = transient ischemic attack.

covariates, although some methodologists would consider 0.20 or less adequate. ${ }^{27}$

Second, event rates were compared between treatment groups using Cox models with inverse probability of treatment weighting (i.e., 6 pairwise comparisons for the 3 NOACs vs. warfarin [reference] and among the 3 NOACs). Proportional hazard assumptions were visually assessed and tested using the product term of drug exposure and time.

After propensity score weighting, we achieved balance on all the measured covariates, as evidenced by MASD $<0.1$, with the exception of PPI use (MASD =0.23). Therefore, we included this covariate in all Cox models.

\section{Sensitivity Analysis}

We examined treatment effects in prespecified subgroups and for the secondary outcome by repeating the analytic steps previously described (Figures 2 and 3). The robustness of our findings was assessed using several sensitivity analyses. First, to account for the potential bias from differential censoring, we conducted an intention-to-treat analysis in which patients were censored only at end of enrollment or death, compared with the "as-treated" approach used in the primary analysis. Second, because multiple comorbid illnesses adversely affect anticoagulation control, we excluded individuals with 7 or more comorbid illnesses.

We controlled for multiplicity by adjusting the $P$ values of the hazard ratios (HRs) for the primary outcome and subgroups using Benjamini-Hochberg false discovery rates. Statistical analyses were conducted with SAS software version 9.4 (SAS Institute, Cary, NC).

\section{Results}

\section{Cohort Characteristics}

A total of 44,057 NVAF patients initiated anticoagulation with warfarin $(n=23,431)$, dabigatran $(n=8,539)$, apixaban $(n=3,689)$, or rivaroxaban $(n=8,398$; Figure 1$)$. Before propensity score weighting, warfarin users were older than dabigatran, apixaban, and rivaroxaban users (mean age: 73 vs. 66 vs. 69 vs. 67 years). The warfarin cohort had the highest proportion of women (warfarin vs. dabigatran vs. apixaban vs. rivaroxaban: $43.5 \%$ vs. $34.5 \%$ vs. $40.9 \%$ vs. $38.9 \%$ ). Mean $\mathrm{CHA}_{2} \mathrm{DS}_{2}$-VASc and HAS-BLED scores were significantly higher in warfarin users at baseline (warfarin vs. dabigatran vs. apixaban vs. rivaroxaban: 3.7 vs. 2.7 vs. 3.2 vs. 2.9 and 2.3 vs. 1.8 vs. 2.1 vs. 1.9 , respectively). Balance was achieved on all baseline characteristics across the treatment groups except the use of PPIs (Table 1).

\section{Bleeding Risk}

Major bleeding events numbered 2,337 during 36,636.2 person-years of follow-up (1,729 warfarin vs. 245 apixaban vs. 62 dabigatran vs. 301 rivaroxaban; Table 2). The number of events, person-years at risk, and crude event rates for all the subgroups are shown in Appendix B (available in online article). 
Major Bleeding Risk During Anticoagulation with Warfarin, Dabigatran,

Apixaban, or Rivaroxaban in Patients with Nonvalvular Atrial Fibrillation

FIGURE 3 Bleeding Events, Entire Cohort and Subgroups, Among NOACs, After Propensity Score Weighting

\begin{tabular}{|c|c|c|c|c|c|c|c|}
\hline \multirow{3}{*}{$\begin{array}{l}\text { Outcome } \\
\text { Major bleeding events }^{a}\end{array}$} & \multicolumn{3}{|c|}{ Dabigatran vs. Apixaban } & \multicolumn{2}{|c|}{ Dabigatran vs. Rivaroxaban } & \multicolumn{2}{|c|}{ Apixaban vs. Rivaroxaban } \\
\hline & \multicolumn{2}{|c|}{$\begin{array}{c}\text { Favors } \\
\text { dabigatran }\end{array}$} & $\begin{array}{l}\text { Favors } \\
\text { apixaban }\end{array}$ & $\begin{array}{c}\text { Favors } \\
\text { dabigatran }\end{array}$ & $\begin{array}{c}\text { Favors } \\
\text { rivaroxaban }\end{array}$ & $\begin{array}{l}\text { Favors } \\
\text { apixaban }\end{array}$ & $\begin{array}{c}\text { Favors } \\
\text { rivaroxaban }\end{array}$ \\
\hline & $\begin{array}{c}1.29 \\
(0.99-1.69) \\
\end{array}$ & & & $\begin{array}{c}0.67 \\
(0.58-0.78)\end{array}$ & & $\begin{array}{cc}0.52 \\
(0.40-0.68)\end{array}$ & \\
\hline Intracranial bleeding & $\begin{array}{c}0.57 \\
(0.33-0.98)^{\mathrm{b}} \\
\end{array}$ & -1 & & $\begin{array}{c}0.54 \\
(0.43-0.96)^{\mathrm{b}}\end{array}$ & & $\begin{array}{c}1.13 \\
(0.66-1.93) \\
\end{array}$ & \\
\hline Major GI bleeding & $\begin{array}{c}1.43 \\
(1.09-1.88) \\
\end{array}$ & & & $\begin{array}{c}1.17 \\
(0.99-1.38) \\
\end{array}$ & - & $\begin{array}{c}0.82 \\
(0.62-1.08) \\
\end{array}$ & \\
\hline \multicolumn{8}{|c|}{ Subgroups (major bleeding events) } \\
\hline \multicolumn{8}{|c|}{ History of bleeding } \\
\hline No & $\begin{array}{c}1.15 \\
(0.86-1.54) \\
\end{array}$ & & & $\begin{array}{c}0.65 \\
(0.55-0.77) \\
\end{array}$ & & $\begin{array}{c}0.56 \\
(0.42-0.75) \\
\end{array}$ & \\
\hline Yes & $\begin{array}{c}2.07 \\
(1.07-4.00)^{\mathrm{b}}\end{array}$ & & $\longrightarrow$ & $\begin{array}{c}0.38 \\
(0.20-0.72) \\
\end{array}$ & & $\begin{array}{c}1.01 \\
(0.79-1.30)\end{array}$ & \\
\hline \multicolumn{8}{|l|}{ Age, years } \\
\hline$<65$ & $\begin{array}{c}1.04 \\
(0.50-2.16) \\
\end{array}$ & 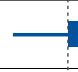 & - & $\begin{array}{c}0.53 \\
(0.43-0.82)\end{array}$ & & $\begin{array}{c}0.51 \\
(0.25-1.02)\end{array}$ & \\
\hline $65-74$ & $\begin{array}{c}0.85 \\
(0.52-1.40) \\
\end{array}$ & - & & $\begin{array}{cc}0.57 & - \\
(0.41-0.78) & - \\
\end{array}$ & & $\begin{array}{c}0.67 \\
(0.41-1.08) \\
\end{array}$ & \\
\hline$\geq 75$ & $\begin{array}{c}1.52 \\
(1.06-2.18)\end{array}$ & & & $\begin{array}{c}0.72 \\
(0.60-0.87) \\
\end{array}$ & & $\begin{array}{c}0.47 \\
(0.33-0.68) \\
\end{array}$ & \\
\hline \multicolumn{8}{|l|}{ HAS-BLED scorec } \\
\hline$\leq 3$ & $\begin{array}{c}1.10 \\
(0.80-1.70) \\
\end{array}$ & & & $\begin{array}{c}0.70 \\
(0.50-0.80) \\
\end{array}$ & & $\begin{array}{c}0.58 \\
(0.40-0.86) \\
\end{array}$ & \\
\hline$\geq 3$ & $\begin{array}{c}1.44 \\
(0.99-2.08)\end{array}$ & & & $\begin{array}{c}0.69 \\
(0.57-0.84) \\
\end{array}$ & & $\begin{array}{c}0.48 \\
(0.33-0.69) \\
\end{array}$ & \\
\hline \multicolumn{8}{|l|}{ Hypertension } \\
\hline No & $\begin{array}{c}1.35 \\
(0.79-2.30) \\
\end{array}$ & & & $\begin{array}{c}0.61 \\
(0.26-0.81) \\
\end{array}$ & & $\begin{array}{c}0.45 \\
(0.27-0.77) \\
\end{array}$ & \\
\hline Yes & $\begin{array}{c}1.28 \\
(0.94-1.74) \\
\end{array}$ & & & $\begin{array}{c}0.70 \\
(0.59-0.84) \\
\end{array}$ & & $\begin{array}{c}0.55 \\
(0.40-0.75) \\
\end{array}$ & \\
\hline \multicolumn{8}{|l|}{ Diabetes mellitus } \\
\hline No & $\begin{array}{c}1.36 \\
(0.98-1.89) \\
\end{array}$ & & & $\begin{array}{c}0.74 \\
(0.62-0.89)\end{array}$ & & $\begin{array}{c}0.55 \\
(0.39-0.76) \\
\end{array}$ & \\
\hline Yes & $\begin{array}{c}1.17 \\
(0.73-1.85) \\
\end{array}$ & & & $\begin{array}{c}0.57 \\
(0.44-0.74)\end{array}$ & & $\begin{array}{c}0.49 \\
(0.31-0.76)\end{array}$ & \\
\hline \multicolumn{8}{|l|}{ Heart failure } \\
\hline No & $\begin{array}{c}1.12 \\
(0.79-1.59) \\
\end{array}$ & 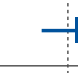 & & $\begin{array}{c}0.61 \\
(0.50-0.74) \\
\end{array}$ & & $\begin{array}{c}0.54 \\
(0.38-0.76) \\
\end{array}$ & \\
\hline Yes & $\begin{array}{c}1.56 \\
(1.03-2.37)^{\mathrm{b}} \\
\end{array}$ & & - & $\begin{array}{c}0.77 \\
(0.61-0.96) \\
\end{array}$ & & $\begin{array}{c}0.49 \\
(0.32-0.75)\end{array}$ & \\
\hline \multicolumn{8}{|l|}{$\mathrm{CHA}_{2} \mathrm{DS}_{2}$-VASc score ${ }^{\mathrm{d}}$} \\
\hline 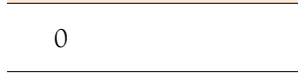 & $\begin{array}{c}0.68 \\
(0.11-4.30) \\
\end{array}$ & +1 & & $\begin{array}{c}1.13 \\
(0.24-5.27) \\
\end{array}$ & $\longrightarrow$ & $\begin{array}{c}1.66 \\
(0.23-12.10) \\
\end{array}$ & - \\
\hline 1 & $\begin{array}{c}1.70 \\
(0.36-8.04) \\
\end{array}$ & & $\longrightarrow$ & $\begin{array}{c}0.37 \\
(0.19-0.75)\end{array}$ & & $\begin{array}{c}0.22 \\
(0.05-0.98)^{\mathrm{b}}-\mathrm{-}\end{array}$ & \\
\hline$\geq 2$ & $\begin{array}{c}1.29 \\
(0.98-1.70) \\
\end{array}$ & & - & $\begin{array}{c}0.69 \\
(0.59-0.81) \\
\end{array}$ & & $\begin{array}{c}0.54 \\
(0.41-0.70) \\
\end{array}$ & \\
\hline \multicolumn{8}{|l|}{ CKD 3 or worse } \\
\hline No & $\begin{array}{c}1.24 \\
(0.92-1.65) \\
\end{array}$ & & -1 & $\begin{array}{c}0.69 \\
(0.59-0.82) \\
\end{array}$ & & $\begin{array}{c}0.56 \\
(0.42-0.75) \\
\end{array}$ & \\
\hline Yes & $\begin{array}{c}1.67 \\
(0.83-3.35) \\
\end{array}$ & 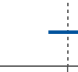 & $\longrightarrow$ & $\begin{array}{c}0.59 \\
(0.41-0.84) \\
\end{array}$ & & $\begin{array}{c}0.35 \\
(0.18-0.70)\end{array}$ & \\
\hline & 0.0 & 1. & 2.0 & 0.0 & 2.0 & 0.0 & 2.0 \\
\hline
\end{tabular}


Major Bleeding Risk During Anticoagulation with Warfarin, Dabigatran,

Apixaban, or Rivaroxaban in Patients with Nonvalvular Atrial Fibrillation

\section{FIGURE 3 Bleeding Events, Entire Cohort and Subgroups, Among NOACs, After Propensity} Score Weighting (continued)

\begin{tabular}{|c|c|c|c|c|c|c|c|}
\hline \multirow[b]{2}{*}{ Outcome } & \multicolumn{2}{|c|}{ Dabigatran vs. Apixaban } & \multicolumn{2}{|c|}{ Dabigatran vs. Rivaroxaban } & \multicolumn{3}{|c|}{ Apixaban vs. Rivaroxaban } \\
\hline & $\begin{array}{c}\text { Favors } \\
\text { dabigatran }\end{array}$ & $\begin{array}{c}\text { Favors } \\
\text { apixaban }\end{array}$ & $\begin{array}{c}\text { Favors } \\
\text { dabigatran }\end{array}$ & $\begin{array}{c}\text { Favors } \\
\text { rivaroxaban }\end{array}$ & $\begin{array}{c}\text { Favors } \\
\text { apixaban }\end{array}$ & \multicolumn{2}{|l|}{$\begin{array}{c}\text { Favors } \\
\text { rivaroxaban }\end{array}$} \\
\hline \multicolumn{8}{|c|}{ Subgroups (major bleeding events) } \\
\hline \multicolumn{8}{|c|}{ NSAIDs } \\
\hline No & $\begin{array}{c}1.22 \\
(0.93-1.61) \\
\end{array}$ & & $\begin{array}{c}0.68 \\
(0.58-0.79) \\
\end{array}$ & & $\begin{array}{c}0.55 \\
(0.42-0.73)\end{array}$ & & \\
\hline Yes & $\begin{array}{c}2.62 \\
(0.82-8.35) \\
\end{array}$ & $\longrightarrow$ & $\begin{array}{c}0.67 \\
(0.41-1.08) \\
\end{array}$ & & $\begin{array}{c}0.26 \\
(0.08-0.81)\end{array}$ & & \\
\hline \multicolumn{8}{|c|}{ Platelet aggragation inhibitors } \\
\hline No & $\begin{array}{c}1.25 \\
(0.93-1.69) \\
\end{array}$ & & $\begin{array}{c}0.69 \\
(0.58-0.81) \\
\end{array}$ & & $\begin{array}{c}0.55 \\
(0.41-0.74)\end{array}$ & & \\
\hline Yes & $\begin{array}{c}1.52 \\
(0.84-2.76)\end{array}$ & & $\begin{array}{c}0.67 \\
(0.49-0.91) \\
\end{array}$ & & $\begin{array}{c}0.44 \\
(0.24-0.79)\end{array}$ & & \\
\hline & 0.0 & 2.0 & 0.0 & 2.0 & 0.0 & 2.0 & 3.0 \\
\hline
\end{tabular}

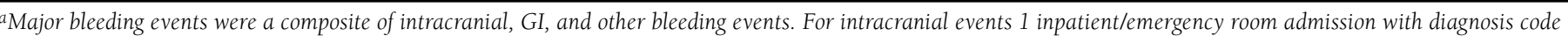
in any position on the claim; for other major bleeding events, primary diagnosis was required.

${ }^{b}$ Not statistically significant after adjustment for multiple comparisons.

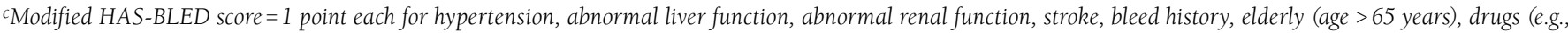

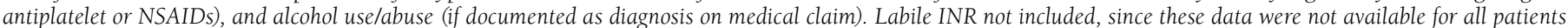

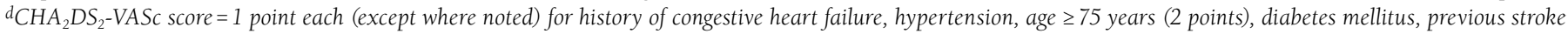
or TIA or thromboembolism (2 points), vascular disease, sex category, and age 65-74 years.

CKD = chronic kidney disease; GI= gastrointestinal; INR=international normalized ratio; NOAC=non-vitamin Koral anticoagulant; NSAID = nonsteroidal anti-inflammatory drug; TIA = transient ischemic attack

Comparing the crude HRs for NOACs versus warfarin and within NOAC comparisons after propensity score weighting, dabigatran ( $\mathrm{HR}=0.67,95 \%$ confidence interval $[\mathrm{CI}]=0.60$ $0.76)$ and apixaban users ( $\mathrm{HR}=0.52,95 \% \mathrm{CI}=0.41-0.67)$ experienced fewer major bleeding events compared with warfarin users. Major bleeding risk was similar between rivaroxaban $(\mathrm{HR}=1.00,95 \% \mathrm{CI}=0.89-1.12)$ and warfarin users. While dabigatran users had a significantly higher risk of major gastrointestinal bleeding ( $\mathrm{HR}=1.17,95 \% \mathrm{CI}=1.04-1.32)$, they experienced significantly fewer intracranial bleeding events $(\mathrm{HR}=0.47,95 \% \mathrm{CI}=0.35-0.65)$, compared with warfarin.

Compared with rivaroxaban users, major bleeding risk was $33 \%$ and $48 \%$ lower in dabigatran and apixaban users, respectively ( $\mathrm{HR}=0.67,95 \% \mathrm{CI}=0.58-0.78$ and $\mathrm{HR}=0.52,95 \%$ $\mathrm{CI}=0.40-0.68$; Figure 3). Relative to apixaban, dabigatran was associated with a significantly higher risk of major gastrointestinal bleeding ( $\mathrm{HR}=1.43,95 \% \mathrm{CI}=1.09-1.88)$ but no difference in overall major bleeding risk. Dabigatran and apixaban users experienced fewer major bleeding events compared with warfarin in almost all of the subgroups examined. No meaningful differences in major bleeding risk exist between warfarin and rivaroxaban users across the subgroups examined (Figure 2).

Across most subgroups of NOAC users that we examined, there was no difference in major bleeding risk with dabigatran and apixaban (Figure 3). However, rivaroxaban users were more likely to experience major bleeding events compared with dabigatran and apixaban (Figure 3).
We conducted sensitivity analyses and found that major bleeding risk among the treatment groups did not considerably change in magnitude or direction.

\section{Discussion}

In this retrospective cohort study of major bleeding risk with anticoagulants for NVAF, we found that, compared with warfarin, dabigatran and apixaban were associated with fewer major bleeding events, which was consistent with the pivotal trials. ${ }^{5-7}$ The rates of major bleeding in this study (2.8\%-6\% per 100 patient-years), while significantly higher than the rates in the pivotal trials, are consistent with recent reports from NOAC registries and likely reflect the difference between a clinical trial population and real-world patients.

The findings in this study are directionally consistent with several recent observational studies that compared bleeding rates associated with NOACs and warfarin and assessed bleeding rates among the newer anticoaulants relative to each other. ${ }^{8,911,12,30}$ In their comparison of major bleeding rates between 3 NOACs and warfarin, Yao et al. reported lower risk for apixaban and dabigatran (apixaban: $\mathrm{HR}=0.45$, 95\% CI $=0.34-0.59, P<0.001 ;$ dabigatran: $H R=0.79,95 \%$ $\mathrm{CI}=0.67-0.94, \quad P<0.01)$; however, rivaroxaban appeared to have similar risk $(\mathrm{HR}=1.04,95 \% \mathrm{CI}=0.90-1.20, P=0.60)$ per 100 patient-years. $^{12}$ 
Major Bleeding Risk During Anticoagulation with Warfarin, Dabigatran,

Apixaban, or Rivaroxaban in Patients with Nonvalvular Atrial Fibrillation

TABLE 1 Baseline Characteristics of Treatment Groups, After Propensity Score Weighting

\begin{tabular}{|c|c|c|c|c|c|}
\hline Characteristic & $\begin{array}{c}\text { Warfarin } \\
(\mathrm{n}=23,431)\end{array}$ & $\begin{array}{c}\text { Dabigatran } \\
(\mathrm{n}=8,539)\end{array}$ & $\begin{array}{l}\text { Apixaban } \\
(\mathrm{n}=3,689)\end{array}$ & $\begin{array}{c}\text { Rivaroxaban } \\
(\mathrm{n}=8,398)\end{array}$ & $\begin{array}{c}\text { Maximum } \\
\text { ASD }^{\mathrm{a}}\end{array}$ \\
\hline \multicolumn{6}{|l|}{ Demographic, \% } \\
\hline Age, years, mean (SD) & $70 \quad(12.2)$ & $70 \quad(12.3)$ & $70 \quad(12.6)$ & $70 \quad(12.3)$ & 0.01 \\
\hline$<65$ & 33.4 & 33.9 & 33.3 & 33.9 & 0.01 \\
\hline $65-74$ & 27.0 & 25.9 & 26.5 & 26.5 & 0.02 \\
\hline$\geq 75$ & 39.6 & 40.2 & 40.2 & 39.6 & 0.01 \\
\hline Female & 40.9 & 41.1 & 40.5 & 41.3 & 0.01 \\
\hline Medicare Advantage & 33.4 & 33.3 & 33.0 & 33.7 & 0.01 \\
\hline \multicolumn{6}{|l|}{ Residence, region } \\
\hline Missing & 1.8 & 1.9 & 2.1 & 1.8 & 0.02 \\
\hline Northeast & 18.8 & 18.7 & 18.9 & 18.7 & 0.01 \\
\hline Midwest & 34.5 & 34.6 & 34.4 & 34.4 & 0.00 \\
\hline West & 20.4 & 20.5 & 19.8 & 20.4 & 0.02 \\
\hline South & 24.6 & 24.3 & 24.8 & 24.8 & 0.01 \\
\hline \multicolumn{6}{|l|}{ Clinical indicators/medication use at baseline, \% } \\
\hline Deyo-Charlson comorbidity score, mean (SD) & $4.4 \quad(2.3)$ & $4.4 \quad(2.3)$ & $4.5 \quad(2.3)$ & $4.4 \quad(2.3)$ & 0.01 \\
\hline HAS-BLED score, ${ }^{\mathrm{b}}$ mean (SD) & $2.1 \quad(1.4)$ & $2.1 \quad(1.4)$ & $2.1 \quad(1.4)$ & $2.1 \quad(1.4)$ & 0.01 \\
\hline $\mathrm{CHA}_{2} \mathrm{DS}_{2}$-VASc score, ${ }^{\mathrm{c}}$ mean $(\mathrm{SD})$ & $3.3(1.8)$ & $3.3(1.9)$ & $3.3 \quad(1.9)$ & $3.3 \quad(1.9)$ & 0.01 \\
\hline Presence of 7 or more comorbidities ${ }^{\mathrm{d}}$ & 9.5 & 10.0 & 9.6 & 9.9 & 0.01 \\
\hline Diabetes mellitus & 28.4 & 28.5 & 29.4 & 28.3 & 0.02 \\
\hline Hypertension & 59.8 & 60.5 & 60.0 & 60.0 & 0.01 \\
\hline Liver disease & 4.7 & 4.5 & 4.6 & 4.7 & 0.01 \\
\hline Congestive heart failure & 27.7 & 28.0 & 28.2 & 28.1 & 0.00 \\
\hline Chronic kidney disease & 10.1 & 10.3 & 10.1 & 10.4 & 0.01 \\
\hline Cerebrovascular disease & 15.9 & 16.2 & 15.6 & 16.2 & 0.02 \\
\hline Coronary heart disease & 36.2 & 36.5 & 36.6 & 36.3 & 0.01 \\
\hline Peripheral vascular disease & 19.9 & 20.1 & 19.8 & 20.1 & 0.01 \\
\hline History of bleeding & 14.9 & 14.5 & 14.3 & 14.2 & 0.02 \\
\hline Helicobacter pylori infection & 0.3 & 0.3 & 0.2 & 0.2 & 0.02 \\
\hline NSAID & 9.8 & 9.5 & 9.7 & 9.9 & 0.01 \\
\hline Platelet aggregation inhibitors & 10.4 & 10.4 & 10.5 & 10.6 & 0.00 \\
\hline Amiodarone & 18.1 & 17.5 & 18.9 & 18.1 & 0.02 \\
\hline Proton pump inhibitors & 34.0 & 35.0 & 24.6 & 28.7 & 0.23 \\
\hline $\begin{array}{l}{ }^{a} \text { Maximum absolute standardized difference consic } \\
{ }^{2} \text { Modified HAS-BLED score }=1 \text { point each for hype } \\
\text { let or NSAIDs), or alcohol excess/abuse. Labile IN } \\
{ }^{c} \mathrm{CH} \mathrm{A}_{2} \mathrm{DS} \mathrm{S}_{2} \text {-VASc score }=1 \text { point each (except wher } \\
\text { or TIA or thromboembolism (2 points), vascular di } \\
\text { dThe number of comorbidities was calculated as th } \\
\text { dementia, gait abnormality, major trauma includir } \\
\text { disease, dyslipidemia, osteoporosis, osteoarthritis o } \\
\text { INR = international normalized ratio; NSAID =nor }\end{array}$ & $\begin{array}{l}\text { wise treatment } \\
\text { ormal liver/renal } \\
\text { hissing for all pat } \\
\text { story of congestiv } \\
\text { egory, and aged } \\
\text { ious history of hy } \\
\text { nemia, coronary } \\
\text { arthritis, and an } \\
\text {-inflammatory d }\end{array}$ & $\begin{array}{l}\text { tion, stroke, blee } \\
\text { since these data } \\
\text { irt failure, hypert } \\
\text { years. } \\
\text { nsion, diabetes } n \\
\text { y disease, cerebr } \\
\text { icer. } \\
S D=\text { standard de }\end{array}$ & $\begin{array}{l}\text { ory, labile INR, } \\
\text { not available for } \\
n, \text { aged } \geq 75 \text { year } \\
\text { s, pericarditis, } d \\
\text { ular disease, con } \\
n ; T I A=\text { transien }\end{array}$ & $\begin{array}{l}\text { ly (aged }>65 \text { year } \\
\text { patients. } \\
\text { points), diabetes r } \\
\text { ess, Parkinson's a } \\
\text { ve heart failure, } p \\
\text { hemic attack. }\end{array}$ & $\begin{array}{l}\text { ugs (e.g., antipl } \\
\text { us, previous strc } \\
\text { eliver disease, } \\
\text { eral vascular }\end{array}$ \\
\hline
\end{tabular}

In an associated study that compared bleeding rates across the newer NOACs, Noseworthy et al. reported that apixaban was associated with less bleeding risk than dabigatran ( $\mathrm{HR}=0.50$, 95\% CI $=0.36-0.70, P<0.001)$ and rivaroxaban $(\mathrm{HR}=0.39,95 \%$ $\mathrm{CI}=0.28-0.54, P<0.001) .{ }^{11}$ Relative to dabigatran, rivaroxaban was associated with increased bleeding risk $(\mathrm{HR}=1.30 ; 95 \%$ $\mathrm{CI}=1.10-1.53, P<0.01) .{ }^{11}$ The study populations evaluated in the Yao et al. and Noseworthy et al. studies most closely approximate the cohorts in our study and were similarly derived from large databases of commercially insured NVAF patients initating
NOACs. ${ }^{11,12}$ Our findings regarding apixaban (i.e., it was associated with lower major bleeding risk compared with warfarin, and among the NOACs evaluated, it appeared to be associated with the lowest risk of major bleeding) are consistent with the findings of these comparable and representative studies.

Also consistent with the results of these studies was our finding that dabigatran appeared to present lower bleeding risks than rivaroxaban and warfarin. We found that the risk of major bleeding was significantly lower across all age categories with dabigatran relative to warfarin, differing from the 
Major Bleeding Risk During Anticoagulation with Warfarin, Dabigatran,

Apixaban, or Rivaroxaban in Patients with Nonvalvular Atrial Fibrillation

TABLE 2 Number of Events, Person-Years at Risk, and Crude Event Rates by Drug Exposure

\begin{tabular}{|c|c|c|c|c|c|c|c|c|c|c|c|c|}
\hline & \multicolumn{3}{|c|}{ Warfarin $(n=23,431)$} & \multicolumn{3}{|c|}{ Dabigatran $(n=8,539)$} & \multicolumn{3}{|c|}{ Apixaban $(n=3,689)$} & \multicolumn{3}{|c|}{ Rivaroxaban $(\mathrm{n}=8,398)$} \\
\hline & $\mathrm{N}$ & PY & Rate & $\mathrm{N}$ & PY & Rate & $\mathrm{N}$ & PY & Rate & $\mathrm{N}$ & PY & Rate \\
\hline Major bleeding & 1,729 & $28,739.6$ & 6.0 & 245 & $8,775.3$ & 2.8 & 62 & $1,893.5$ & 3.3 & 301 & $6,003.10$ & 5.00 \\
\hline Median follow-up, days (IQR) & \multicolumn{3}{|c|}{$285(80-714)$} & \multicolumn{3}{|c|}{$212(68-572)$} & \multicolumn{3}{|c|}{$139(62-271)$} & \multicolumn{3}{|c|}{$169(63-382)$} \\
\hline Major GI bleeding & 649 & $29,623.4$ & 2.2 & 124 & $8,848.0$ & 1.4 & 29 & $1,905.6$ & 0.9 & 92 & $6,083.30$ & 1.50 \\
\hline Intracranial bleeding & 338 & $29,959.0$ & 1.1 & 37 & $8,878.1$ & 0.4 & 14 & $1,907.0$ & 0.7 & 46 & $6,104.61$ & 0.75 \\
\hline
\end{tabular}

$G I=$ gastrointestinal; $I Q R=$ interquartile range; $P Y=$ person-years

RE-LY trial, ${ }^{31}$ which reported a higher major bleeding risk rate in patients aged 75 years or older, although the risk was not significant. ${ }^{5}$ While the overall risk of major bleeding, including intracranial bleeding, was lower with dabigatran compared with warfarin, this safety benefit was attenuated by a higher risk of major gastrointestinal bleeding, a pattern of bleeding first reported in the RE-LY trial and in recent observational studies, ${ }^{5,14,30,31}$

Apixaban was associated with fewer major bleeds compared with warfarin across the subgroups examined, which is consistent with findings from the ARISTOLE trial. Although not examined in this study, a recent reanalysis of ARISTOTLE data suggested that apixaban has a lower risk of major bleeding regardless of dosage adjustment in higher risk groups such as the elderly and individuals with renal impairment. ${ }^{6}$

There were no differences in major bleeding risk between rivaroxaban and warfarin in the entire cohort and subgroups, which is consistent with the results of the ROCKET-AF trial and several other recent observational studies. ${ }^{7,15,32,33}$ The finding of a persistently higher major bleeding risk with rivaroxaban compared with apixaban across subgroups has important implications for patients, providers, and payers and warrants further investigation in randomized clinical trials.

A strength of this study lies in the assembling of a large new user cohort, which allowed us to examine important subgroups that had limited enrollment in the pivotal trials (e.g., elderly individuals, aged $\geq 75$ years, and patients with renal dysfunction). Also, the use of propensity score weighting enabled excellent control of measured baseline differences, and several sensitivity analyses were used to examine the robustness of the findings.

\section{Limitations}

This study has some important limitations to consider. First, although we systematically identified potential confounders in estimating the propensity score weights, our assessment of balance achieved between the treatment groups was limited to the covariates measured. For example, other risk-modifying exposures, such as over-the-counter products (e.g., aspirin, NSAIDs, and PPIs), are not fully captured in administrative claims data. Although there is no reason to believe such exposure misclassification is differentially distributed between the cohorts, residual and unmeasured confounding cannot be excluded. It is also possible that residual confounding arising from baseline differences such as age and other potential confounders may persist despite excellent statistical adjustments.

Second, because the risk of major bleeding is higher in the early phase of anticoagulant therapy, the longer follow-up in the warfarin cohort may disproportionately reflect the anticoagulation experience of patients who continue treatment beyond the early phase of therapy.

Finally, we could not assess the quality of anticoagulation in the warfarin arm or measure the magnitude of this possible confounder. It is possible that patients who are likely to be noncompliant are not selected for anticoagulation with warfarin. Although this limitation is an important one to note, the purpose of this study was to evaluate "real-world" outcomes, so-given that real-world anticoagulation control may be suboptimal - the results of this study need not be misconstrued as nonrepresentative.

\section{Conclusions}

The approval of NOAC medications in recent years provided a promise of less complicated management of anticoagulation compared with warfarin. However, many patients are not treated because of concerns of major bleeding and limited experience with NOACs. In patients treated with warfarin, optimal anticoagulation control is often problematic. In part because of these challenges, anticoagulation for stroke prophylaxis is underused in patients with NVAF who would otherwise benefit. ${ }^{34,35}$ The significantly lower risk of overall major bleeding and major gastrointestinal bleeding observed with apixaban in this study provides evidence of its safety as a suitable warfarin alternative in NVAF patients in a real-world setting, especially for those who have difficulty achieving and maintaining optimal anticoagulation. 


\section{Authors}

GBOYEGA ADEBOYEJE, MD, MS; GOSIA SYLWESTRZAK, MA; and JOHN J. BARRON, PharmD, HealthCore, Wilmington, Delaware. JEFF WHITE, PharmD, MS; ALAN ROSENBERG, MD; JACOB ABARCA, PharmD, MS; and GEOFFREY CRAWFORD, $M D, M S$, Anthem, Indianapolis, Indiana. RITA REDBERG, MD, MSc, University of California, San Francisco.

AUTHOR CORRESPONDENCE: John Barron, PharmD, HealthCore, 123 Justison St., Ste. 200, Wilmington, DE 19801. Tel.: 302.230.2113; E-mail: jbarron@healthcore.com.

\section{DISCLOSURES}

This study was funded by Anthem. Adeboyeje, Sylwestrzak, and Barron are employees of HealthCore, a wholly owned and independently operated subsidiary of Anthem. White, Rosenberg, Abarca, and Crawford are employees of Anthem.

Study concept and design were primarily contributed by Adeboyeje and Sylwestrzak, along with the other authors. Adeboyeje took the lead in data collection, along with Sylwestrzak and Barron. Data interpretation was performed primarily by Rosenberg, Crawford, and Redberg, with assistance from the other authors. The manuscript was written by all the authors and revised primarily by White, Abarca, and Redberg, along with the other authors.

\section{REFERENCES}

1. Hart RG, Pearce LA, Rothbart RM, McAnulty JH, Asinger RW, Halperin JL. Stroke with intermittent atrial fibrillation: incidence and predictors during aspirin therapy. Stroke Prevention in Atrial Fibrillation Investigators. J Am Coll Cardiol. 2000;35(1):183-87.

2. Birman-Deych E, Radford MJ, Nilasena DS, Gage BF. Use and effectiveness of warfarin in Medicare beneficiaries with atrial fibrillation. Stroke. 2006;37(4):1070-74.

3. Hanley CM, Kowey PR. Are the novel anticoagulants better than warfarin for patients with atrial fibrillation? J Thorac Dis. 2015;7(2):165-71.

4. Hylek EM, Evans-Molina C, Shea C, Henault LE, Regan S. Major hemorrhage and tolerability of warfarin in the first year of therapy among elderly patients with atrial fibrillation. Circulation. 2007;115(21):2689-96.

5. Connolly SJ, Ezekowitz MD, Yusuf S, et al. Dabigatran versus warfarin in patients with atrial fibrillation. N Engl J Med. 2009;361(12):1139-51.

6. Granger CB, Alexander JH, McMurray JJ, et al. Apixaban versus warfarin in patients with atrial fibrillation. N Engl J Med. 2011;365(11):981-92.

7. Patel MR, Mahaffey KW, Garg J, et al. Rivaroxaban versus warfarin in nonvalvular atrial fibrillation. N Engl J Med. 2011;365(10):883-91.

8. Sussman M, Ghate S, Sutherland S, et al. Resource use among nonvalvular atrial fibrillation patients. Am J Pharm Benefits. 2016;8(5):84-92.

9. Deitelzweig S, Bruno A, Trocio J, et al. An early evaluation of bleedingrelated hospital readmissions among hospitalized patients with nonvalvular atrial fibrillation treated with direct oral anticoagulants. Curr Med Res Opin. 2016;32(3):573-82.

10. Graham DJ, Reichman ME, Wernecke M, et al. Stroke, bleeding, and mortality risks in elderly medicare beneficiaries treated with dabigatran or rivaroxaban for nonvalvular atrial fibrillation. JAMA Intern Med. 2016;176(11):1662-71.

11. Noseworthy PA, Yao X, Abraham NS, Sangaralingham LR, McBane RD, Shah ND. Direct comparison of dabigatran, rivaroxaban, and apixaban for effectiveness and safety in nonvalvular atrial fibrillation. Chest. 2016;150(6):1302-12.
12. Yao X, Abraham NS, Sangaralingham LR, et al. Effectiveness and safety of dabigatran, rivaroxaban, and apixaban versus warfarin in nonvalvular atrial fibrillation. J Am Heart Assoc. 2016;5(6):e003725. Available at: https:// www.ncbi.nlm.nih.gov/pmc/articles/PMC4937291/. Accessed July 6, 2017.

13. Desai NR, Krumme AA, Schneeweiss S, et al. Patterns of initiation of oral anticoagulants in patients with atrial fibrillation-quality and cost implications. Am J Med. 2014;127(11):1075-82.el.

14. Hernandez I, Baik SH, Pinera A, Zhang Y. Risk of bleeding with dabigatran in atrial fibrillation. JAMA Intern Med. 2015;175(1):18-24.

15. Chang HY, Zhou M, Tang W, Alexander GC, Singh S. Risk of gastrointestinal bleeding associated with oral anticoagulants: population based retrospective cohort study. BMJ. 2015;350:h1585.

16. Pollack CV, Jr., Reilly PA, Eikelboom J, et al. Idarucizumab for dabigatran reversal. N Engl J Med. 2015;373(6):511-20.

17. Ansell JE, Bakhru SH, Laulicht BE, et al. Use of PER977 to reverse the anticoagulant effect of edoxaban. N Engl J Med. 2014;371(22):2141-42.

18. Siegal DM, Curnutte JT, Connolly SJ, et al. Andexanet alfa for the reversal of factor Xa inhibitor activity. N Engl J Med. 2015;373(25):2413-24.

19. Abraham NS, Hartman C, Richardson P, Castillo D, Street RL Jr, Naik AD. Risk of lower and upper gastrointestinal bleeding, transfusions, and hospitalizations with complex antithrombotic therapy in elderly patients. Circulation. 2013;128(17):1869-77.

20. Arnason T, Wells PS, van Walraven C, Forster AJ. Accuracy of coding for possible warfarin complications in hospital discharge abstracts. Thromb Res. 2006;118(2):253-62.

21. Cunningham A, Stein CM, Chung CP, Daugherty JR, Smalley WE, Ray WA. An automated database case definition for serious bleeding related to oral anticoagulant use. Pharmacoepidemiol Drug Saf. 2011;20(6):560-66.

22. Wahl PM, Rodgers K, Schneeweiss S, et al. Validation of claims-based diagnostic and procedure codes for cardiovascular and gastrointestinal serious adverse events in a commercially-insured population. Pharmacoepidemiol Drug Saf. 2010;19(6):596-603.

23. Quan H, Li B, Couris CM, et al. Updating and validating the Charlson Comorbidity Index and score for risk adjustment in hospital discharge abstracts using data from 6 countries. Am J Epidemiol. 2011;173(6):676-82.

24. Lip GY, Nieuwlaat R, Pisters R, Lane DA, Crijns HJ. Refining clinical risk stratification for predicting stroke and thromboembolism in atrial fibrillation using a novel risk factor-based approach: the Euro Heart Survey on Atrial Fibrillation. Chest. 2010;137(2):263-72.

25. Apostolakis S, Lane DA, Guo Y, Buller H, Lip GY. Performance of the HEMORR(2)HAGES, ATRIA, and HAS-BLED bleeding risk-prediction scores in patients with atrial fibrillation undergoing anticoagulation: the AMADEUS (evaluating the use of SR34006 compared to warfarin or acenocoumarol in patients with atrial fibrillation) study. J Am Coll Cardiol. 2012;60(9):861-67.

26. Austin PC. The performance of different propensity score methods for estimating marginal hazard ratios. Stat Med. 2013;32(16):2837-49.

27. McCaffrey DF, Griffin BA, Almirall D, Slaughter ME, Ramchand R, Burgette LF. A tutorial on propensity score estimation for multiple treatments using generalized boosted models. Stat Med. 2013;32(19):3388-414.

28. Feng P, Zhou XH, Zou QM, Fan MY, Li XS. Generalized propensity score for estimating the average treatment effect of multiple treatments. Stat Med. 2012;31(7):681-97.

29. Spreeuwenberg MD, Bartak A, Croon MA, et al. The multiple propensity score as control for bias in the comparison of more than two treatment arms: an introduction from a case study in mental health. Med Care. 2010;48(2):166-74.

30. Graham DJ, Reichman ME, Wernecke M, et al. Cardiovascular, bleeding, and mortality risks in elderly Medicare patients treated with dabigatran or warfarin for nonvalvular atrial fibrillation. Circulation. 2015;131(2):157-64. 
31. Eikelboom JW, Wallentin L, Connolly SJ, et al. Risk of bleeding with 2 doses of dabigatran compared with warfarin in older and younger patients with atrial fibrillation: an analysis of the randomized evaluation of long-term anticoagulant therapy (RE-LY) trial. Circulation. 2011;123(21):2363-72.

32. Maura G, Blotiere PO, Bouillon K, et al. Comparison of the short-term risk of bleeding and arterial thromboembolic events in nonvalvular atrial fibrillation patients newly treated with dabigatran or rivaroxaban versus vitamin K antagonists: a French nationwide propensity-matched cohort study. Circulation. 2015;132(13):1252-60.
33. Sherid M, Sifuentes H, Sulaiman S, et al. Risk of gastrointestinal bleeding with dabigatran: a head-to-head comparative study with rivaroxaban. Digestion. 2014;90(2):137-46.

34. Deitelzweig S. Practical considerations in the use of novel oral anticoagulants for stroke prevention in nonvalvular atrial fibrillation. Cardiovasc Ther. 2014;32(2):74-81.

35. Merli G, Weitz HH. The decision to anticoagulate: assessing whether benefits outweigh the risks for patients with atrial fibrillation. Clin Cardiol. 2004;27(6):313-20. 
Major Bleeding Risk During Anticoagulation with Warfarin, Dabigatran,

Apixaban, or Rivaroxaban in Patients with Nonvalvular Atrial Fibrillation

\section{APPENDIX A Covariate Definitions}

Covariate

Age

Male

Medicare supplemental

Medicare Advantage

Region of residence

Indexes

Comorbidity index

$\mathrm{CHA}_{2} \mathrm{DS}_{2}$-VASc score

HAS-BLED score

Comorbid illnesses

Ischemic stroke

Hemorrhagic stroke

Transient ischemic attack

Renal insufficiency

Myocardial infarction

Congestive heart failure

Cerebrovascular disease

Coronary artery disease

Peripheral vascular disease

Cancer

Diabetes mellitus

Hypertension

Dyslipidemia

Pericarditis

Hyperthyroidism

Coagulation defect factors

Dementia

Parkinson's disease

Gait abnormality

Dizziness

Diabetic and alcoholic neuropathy

Esophageal varices

Major trauma
Patient characteristics

Osteoarthritis

Definition

Coding

Age in years

Male sex

Enrollment in a private health insurance in addition to Medicare

Managed health care - PPO or HMO

Region of residence at index date

Deyo-Charlson Comorbidity Index

Congestive heart failure, hypertension, age $\geq 75$ years, diabetes mellitus, previous stroke or TIA or thromboembolism, vascular disease, age 65-74 years, sex category (female gender) Hypertension, abnormal liver/renal function, stroke, bleeding history, labile INR, elderly (age > 65), drugs (e.g., antiplatelet or NSAIDs) or alcohol concomitantly (1 point each). Labile INR set to missing for all patients

ICD-9-CM codes 433.01, 433.10 , 433.11, 433.21, 433.31, 433.81, 433.91, 434.00, 434.01, 434.11, 434.91, 436 ICD 9-CM codes 430. $\mathrm{x}-432 . \mathrm{x}$ ICD-9-CM code 435.xx ICD-9-CM codes 582.xx, 585.xx, 588.xx

ICD-9-CM code 410.xx ICD-9-CM codes 402.01, 402.11, 402.91, 404.01, 404.03, 404.11, 404.13, 404.91, 404.93, 428.xx

ICD-9-CM codes 430. $x-435 . x$ ICD-9-CM codes 410.xx, 411.1x, 411.8x, 412.xx, 413.xx, 414.0x, 414.2x, 414.3x

ICD-9-CM codes 433.xx, 437.0x, 437.1x, 440.xx, 443.xx ICD-9-CM codes 140.xx-239.xx, V10.xx

ICD-9-CM codes 715.xx, 721.x

ICD-9-CM codes 250.xx AND/OR use of oral glucose-lowering medications (GPI starts with 27, except 2710 and 2730)

ICD-9-CM codes 401.xx, 402.xx, 403.xx, 404.xx

ICD-9-CM code 272.xx OR GPI starts with 39

ICD-9-CM code 420.xx

ICD-9-CM codes $242.0 \mathrm{x}-242.9 \mathrm{x}$

ICD-9-CM codes 286.1x, 286.3x, 286.5x, 285.5x, 286.5x, $286.4 x, 286.4 x, 286.3 x, 286.5 x, 286.5 x, 287.4 x, 287.5 x$, $287.8 \mathrm{x}, 270.4 \mathrm{x}$

ICD-9-CM codes 290.xx, 294.xx, 330.xx, 331.xx

ICD-9-CM code 332.xx

ICD-9-CM codes 334.xx, 781.2x

ICD-9-CM codes $458.0 \times, 780.4 \times$

ICD-9-CM codes $357.2 \mathrm{x}$ and/or $357.5 \mathrm{x}$

ICD-9-CM codes 456.0 $\mathrm{x}-456.2 \mathrm{x}$

ICD-9-CM codes 806.xx, 808.xx, 809.xx, 820.xx, 821.xx, 822.xx, 823.xx, 824.xx, 825.xx, 826.xx, 827.xx, 828.xx 829.xx, 835.xx, 843.xx, 901.1x, 901.2x, 901.3x, 901.83, $902.0 x, 902.1 x, 902.5 x, 902.51,902.52,902.53,902.54$ 902.59, 902.87, 903.0x, 903.1x, 904.0x, 904.1x, 904.2x 904.4x, 904.5x, 924.xx, 928.xx, 942.xx, 943.xx, 945.xx, 952.xx, 959.6x, 959.7x, 897.xx, 820.xx-929.xx

Pre-index medications

Antiarrhymics

Any pharmacy claim

Amiodarone

Any pharmacy claim

Diuretics
Continuous

Dichotomous (yes/no)

Dichotomous (yes/no)

Dichotomous (yes/no)

Categorical (Northeast, Midwest, West, South, missing)

Interval (0-33)

(1 point each, 2 points for age $\geq 75$ years and stroke for a maximum of 9)

(1 point each for a maximum of 8 )

Dichotomous (yes/no)

Dichotomous (yes/no)

Dichotomous (yes/no)

Dichotomous (yes/no)

Dichotomous (yes/no)

Dichotomous (yes/no)

Dichotomous (yes/no)

Dichotomous (yes/no)

Dichotomous (yes/no)

Dichotomous (yes/no)

Dichotomous (yes/no)

Dichotomous (yes/no)

Dichotomous (yes/no)

Dichotomous (yes/no)

Dichotomous (yes/no)

Dichotomous (yes/no)

Dichotomous (yes/no)

Dichotomous (yes/no)

Dichotomous (yes/no)

Dichotomous (yes/no)

Dichotomous (yes/no)

Dichotomous (yes/no)

Dichotomous (yes/no)

Dichotomous (yes/no)

Dichotomous (yes/no)

Dichotomous (yes/no) 
Major Bleeding Risk During Anticoagulation with Warfarin, Dabigatran,

Apixaban, or Rivaroxaban in Patients with Nonvalvular Atrial Fibrillation

\section{APPENDIX A Covariate Definitions (continued)}

Covariate

Definition

Coding

Pre-index medications

Vasopressors

Antihyperlipidemics

COX-2 inhibitors

Platelet aggregation inhibitors

Anti-inflammatory agents

Proton pump inhibitor

Stroke

Any pharmacy claim

Any pharmacy claim

Any pharmacy claim

Any pharmacy claim

Any pharmacy claim

Any pharmacy claim

Any pharmacy claim

ICD-9-CM codes for ischemic (433.01, 433.10, 433.11, 433.21, Dichotomous (yes/no)

433.31, 433.81, 433.91, 434.00, 434.01, 434.11 , 434.91, 436)

or hemorrhagic stroke (430.x-432.x)

Arterial embolism and thrombosis

ICD-9-CM codes for 444.xx

Dichotomous (yes/no)

Venous thromboembolism (VTE)

Inpatient or emergency department ICD-9-CM codes for deep

venous thromboembolism (451.1x, 451.2x, 451.81, 451.83,

$451.84,451.9 x, 453.1 x, 453.2 x, 453.4 x, 453.5 x, 453.6 x$,

453.7x, 453.8x, 453.9x, 997.2x) or pulmonary embolism (415.1x)

Major bleeding

ICD-9-CM codes 423.0x, 430.xx, 431xx, 432xx, 852.0x,

$852.2 \mathrm{x}, 852.4 \mathrm{x}, 853.0 \mathrm{x}, 455.2,455.5,455.8,456.0,456.20$,

$459.0 x, 530.7 x, 530.82,531.00,531.01,531.20,531.21$,

$531.40,531.41,531.60,531.61,533.01,533.20,533.21$,

$533.40,533.41,533.60,533.61,534.00,534.01,534.20$,

$534.21,534.40,534.41,534.60,534.61,535.11,535.21$,

$535.31,535.41,535.51,535.61,537.83,562.02,562.03$

$562.12,562.13,568.81,569.3,569.85,578,578.0,578.1$,

578.9, 593.81, 599.7, 719.10, 719.11, 719.12, 719.13, 719.14,

719.15, 719.16, 719.17, 719.18, 719.19, 784.7, 784.8x, 786.3x

1 inpatient claim with ICD-9-CM diagnosis codes 430.xx, 431.xx, 432.xx, 852.0x, 852.2x, 852.4x, 853.0x in any position of the claim and the remaining ICD-9-CM codes in the primary position (primary diagnosis) of the claim

Exclusions

ICD-9-CM codes 394.0x , 394.1x, 394.2x, 394.9x, 396.xx

V43.3x, Z95.2x, V42.2x, 238.4x, 238.71, 283.2x, 289.81, 289.91, 5855, 5856

Kidney transplant

ICD-9-CM codes V42.0x, 996.81 or ICD-9-CM procedure codes 55.6x or CPT codes 50340, 50360, 50365, 50370, 50380

Chronic dialysis

ICD-9-CM codes 792.5x, V56.2x or ICD-9-CM procedure codes $39.95,54.98$ or CPT codes $90935,90937,90945$, 90947, 99512, 99601, 99602

CPT = Current Procedural Terminology; GPI = Generic Product Identifier; ICD-9-CM = International Classification of Diseases, Ninth Revision, Clinical Modification; $I N R=$ international normalized ratio; NSAID = nonsteroidal anti-inflammatory drug; TIA = transient ischemic attack . 
Major Bleeding Risk During Anticoagulation with Warfarin, Dabigatran,

Apixaban, or Rivaroxaban in Patients with Nonvalvular Atrial Fibrillation

APPENDIX B Number of Events, Person-Years at Risk, and Crude Event Rates of Major Bleeding Events

\begin{tabular}{|c|c|c|c|c|c|c|c|c|c|c|c|c|}
\hline \multirow{2}{*}{$\begin{array}{l}\text { Major Bleeding } \\
\text { Subgroups }\end{array}$} & \multicolumn{3}{|c|}{ Warfarin } & \multicolumn{3}{|c|}{ Dabigatran } & \multicolumn{3}{|c|}{ Apixaban } & \multicolumn{3}{|c|}{ Rivaroxaban } \\
\hline & $\mathrm{N}$ & PYs & Rate & $\mathrm{N}$ & PYs & Rate & $\mathrm{N}$ & PYs & Rate & $\mathrm{N}$ & PYs & Rate \\
\hline \multicolumn{13}{|c|}{ History of bleeding } \\
\hline No & 1,328 & $24,111.3$ & 5.51 & 195 & $7,817.1$ & 2.49 & 51 & $1,635.3$ & 3.12 & 243 & $5,315.1$ & 4.57 \\
\hline Yes & 401 & $4,628.3$ & 8.66 & 50 & 958.2 & 5.22 & 11 & 258.2 & 4.26 & 11 & 258.2 & 4.26 \\
\hline \multicolumn{13}{|c|}{ Age categories, years } \\
\hline$<65$ & 195 & $5,273.5$ & 3.70 & 44 & $3,659.2$ & 1.20 & 11 & 716.2 & 1.54 & 62 & $2,423.3$ & 2.56 \\
\hline $65-74$ & 433 & $8,477.4$ & 5.11 & 59 & $2,421.8$ & 2.44 & 18 & 485.3 & 3.71 & 80 & $1,678.5$ & 4.77 \\
\hline$\geq 75$ & 1,101 & $14,988.7$ & 7.35 & 142 & $2,694.3$ & 5.27 & 33 & 692.0 & 4.77 & 159 & $1,901.3$ & 8.36 \\
\hline \multicolumn{13}{|l|}{ Hypertension } \\
\hline No & 550 & $11,334.2$ & 4.85 & 73 & $3,665.8$ & 1.99 & 14 & 652.3 & 2.15 & 85 & $2,338.3$ & 3.64 \\
\hline Yes & 1,179 & $17,405.4$ & 6.77 & 172 & $5,109.6$ & 3.37 & 48 & $1,241.2$ & 3.87 & 216 & $3,664.8$ & 5.89 \\
\hline \multicolumn{13}{|l|}{ Diabetes mellitus } \\
\hline No & 1,133 & $19,764.9$ & 5.73 & 181 & $6,538.3$ & 2.77 & 42 & $1,413.5$ & 2.97 & 204 & $4,436.6$ & 4.60 \\
\hline Yes & 596 & $8,974.7$ & 6.64 & 64 & $2,237.0$ & 2.86 & 20 & 480.0 & 4.17 & 97 & $1,566.5$ & 6.19 \\
\hline \multicolumn{13}{|l|}{ Heart failure } \\
\hline No & 1,041 & $20,172.2$ & 5.16 & 154 & $6,866.3$ & 2.24 & 40 & $1,455.4$ & 2.75 & 196 & $4,691.4$ & 4.18 \\
\hline Yes & 688 & $8,567.4$ & 8.03 & 91 & $1,909.0$ & 4.77 & 22 & 438.1 & 5.02 & 105 & $1,311.6$ & 8.01 \\
\hline \multicolumn{13}{|c|}{$\mathrm{CHA}_{2} \mathrm{DS}_{2}$-VASc score ${ }^{\mathrm{a}}$} \\
\hline 0 & 19 & 799.1 & 2.38 & 7 & 734.9 & 0.95 & 2 & 109.8 & 1.82 & 4 & 404.2 & 0.99 \\
\hline 1 & 67 & $2,042.0$ & 3.28 & 16 & $1,370.3$ & 1.17 & 3 & 271.0 & 1.11 & 29 & 931.1 & 3.11 \\
\hline$\geq 2$ & 1,643 & $25,897.5$ & 6.34 & 222 & $6,670.2$ & 3.33 & 57 & $1,512.6$ & 3.77 & 268 & $4,667.7$ & 5.74 \\
\hline \multicolumn{13}{|c|}{ Previous stroke or TIA } \\
\hline No & 1,443 & $25,308.7$ & 5.70 & 208 & $7,933.0$ & 2.62 & 48 & $1,680.1$ & 2.86 & 250 & $5,375.5$ & 4.65 \\
\hline Yes & 286 & $3,431.0$ & 8.34 & 37 & 842.3 & 4.39 & 14 & 213.4 & 6.56 & 51 & 627.6 & 8.13 \\
\hline \multicolumn{13}{|l|}{ CKD 3 or worse } \\
\hline No & 1,420 & $25,717.5$ & 5.52 & 222 & $8,348.5$ & 2.66 & 53 & $1,728.2$ & 3.07 & 259 & $5,685.3$ & 4.56 \\
\hline Yes & 309 & $3,022.1$ & 10.2 & 23 & 426.8 & 5.39 & 9 & 165.2 & 5.45 & 42 & 317.8 & 13.2 \\
\hline \multicolumn{13}{|c|}{ HAS-BLED score ${ }^{b}$} \\
\hline$<3$ & 840 & $17,993.0$ & 4.67 & 124 & $6,384.9$ & 1.94 & 29 & $1,262.0$ & 2.30 & 145 & $4,212.5$ & 3.44 \\
\hline$\geq 3$ & 889 & $10,746.6$ & 8.27 & 121 & $2,390.4$ & 5.06 & 33 & 631.4 & 5.23 & 156 & $1,790.6$ & 8.71 \\
\hline \multicolumn{13}{|l|}{ NSAID } \\
\hline No & 1,601 & $26,416.9$ & 6.06 & 218 & $7,846.6$ & 2.78 & 58 & $1,687.6$ & 3.44 & 266 & $5,325.5$ & 4.99 \\
\hline Yes & 128 & $2,322.7$ & 55.1 & 27 & 928.8 & 2.91 & 4 & 205.8 & 1.94 & 35 & 677.6 & 5.17 \\
\hline \multicolumn{13}{|c|}{ Platelet aggregation inhibitors } \\
\hline No & 1,467 & $26,066.5$ & 5.63 & 197 & $7,914.2$ & 2.49 & 49 & $1,662.2$ & 2.95 & 240 & $5,374.0$ & 4.47 \\
\hline Yes & 262 & 2673.1 & 9.80 & 48 & 861.1 & 5.57 & 13 & 231.3 & 5.62 & 61 & 629.1 & 9.70 \\
\hline \multicolumn{13}{|c|}{$\begin{array}{l}{ }^{a} C H A_{2} D S_{2}-V A S c \text { score }=1 \text { point each (except where noted) for history of congestive heart failure, hypertension, age } \geq 75 \text { years (2 points), diabetes mellitus, previous stroke } \\
\text { or TIA or thromboembolism ( } 2 \text { points), vascular disease, sex category, and age } 65-74 \text { years. } \\
\text { bModified HAS-BLED }=1 \text { point each for hypertension, abnormal liver function, abnormal renal function, stroke, bleed history, elderly (age }>65 \text { years), drugs (e.g., } \\
\text { antiplatelet or NSAIDs), and alcohol uselabuse (if documented as diagnosis on medical claim). Labile INR not included, since these data were not available for all patients. } \\
\text { CKD = chronic kidney disease; INR = international normalized ratio; NSAID = nonsteroidal anti-inflammatory drug; PY=person-years; TIA = transient ischemic attack. }\end{array}$} \\
\hline
\end{tabular}

